\title{
Multiple Metastatic Small Cell Carcinoma of Unknown Primary Diagnosed on Inguinal Node Biopsy
}

\author{
${ }^{*}$ Capizzello Antonio ${ }^{1}$, Tsanadis Konstantinos ${ }^{1}$, Iliadis Alexandros ${ }^{2}$, Kostopoulos Ioannis ${ }^{2}$ and Tzitzikas \\ Ioannis $^{1}$ \\ ${ }^{1}$ Department of Radiation Oncology of University Hospital AHEPA, Thessaloniki, Greece \\ ${ }^{2}$ Department of Pathology, Faculty of Medicine, Aristotle University, Thessaloniki, Greece \\ Submission: April 28, 2016; Published: May 16, 2016 \\ "Corresponding author: Capizzello Antonio, AHEPA University Hospital of Thessaloniki, Stilponos Kyriakidi 1, Thessaloniki, 54636, Greece , Tel: \\ 00302310993421; Email: antoniocapi@msn.com
}

\begin{abstract}
Poorly differentiated neuroendocrine tumors are uncommon neoplasms of the lung and the gastrointestinal tract. However, 3-5\% of these tumors are diagnosed as metastatic disease with unknown primary. Here, we describe a case of neuroendocrine small cell carcinoma of unknown primary diagnosed by inguinal node biopsy. A 53-year-old male patient with a history of heavy smoking presented with cough, back pain and altered mental status. Whole body imaging studies (CT - MRI - FDG PET) favored a diagnosis of hematological disease (lymphoma) or diffuse metastatic melanoma. However, inguinal node biopsy revealed morphological and immunohistochemical features of a neuroendocrine carcinoma, probably originating from the lung. The patient received palliative whole brain irradiation and etoposide/platinum-based chemotherapy.
\end{abstract}

Abbreviations: CUP: Cancer of Unknown Primary; IHC: Immunohistochemistry; CT: Computed Tomography; MRI: Magnetic Resonance Imaging; PET: Positron Emission Tomography

\section{Introduction}

Metastatic cancer of unknown primary (CUP) accounts for $3-5 \%$ of all malignant neoplasms. International registries from seven countries have reported incidences ranging from $2.3 \%$ to $7.8 \%$ [1]. More than $50 \%$ of CUP patients present with multiple sites of involvement, while the rest have a single site. The progression of such cancer with unknown origin is rapid in most cases and shows atypical metastatic patterns. Most frequent locations of dominant metastatic sites are mediastinum, retroperitoneum and peripheral lymph nodes [2]. CUP is also more common in lymph nodes of the neck and axillary regions, while inguinal involvement accounts for $<5 \%$ of cases [3]. Since CUP in the inguinal region is rare, there is a paucity of literature on the management of such patients.

Neuroendocrine carcinomas with widely varying clinical and histologic features account for approximately 3\% of all CUP [4]. Neuroendocrine cells are distributed widely throughout the body and neoplasms of these dispersed cells can occur at many sites. Of major importance is the classification of this group into tumors with low-grade histology (classic carcinoid) and indolent clinical course vs those with high-grade histology (small or large cell with neuroendocrine features) and an aggressive clinical course [5]. The origin of these high-grade neuroendocrine carcinomas remains unclear. Panels of immunohistochemical (IHC) stains or molecular gene expression tumor profiling are used for the determination of the tissue of origin in CUP patients [6]. CUP of the neuroendocrine type may have arisen from an occult extrapulmonary site, but are correctly described as neuroendocrine car $\neg$ cinoma of an unknown primary site. Patients with small cell carcinoma at a metastatic site are considered for systemic therapy at the time of diagnosis. Although the optimum regimen is undefined, a platinum/etoposide-based regimen is currently used [7]. In this case report, we describe an uncommon case of inguinal lymph node small cell metastasis of unknown origin, accompanied by multiple sites of involvement.

\section{Case Presentation}

A 53-year-old male, with a history of heavy smoking, presented with polyuria, polydipsia and episodes of transient memory loss. He suffered from back pain, night cough, cyanosis and an altered mental status. There was no history 


\section{Cancer Therapy \& Oncology International Journal}

of fever or night sweats. On clinical examination, there were multiple palpable subcutaneous nodules on the trunk and tenderly swollen inguinal lymph nodes. The remainder of clinical examination and initial laboratory investigations had normal findings. The results of laboratory examinations, including urinalysis, liver and renal function tests, revealed no abnormalities. Upper and lower gastrointestinal endoscopy was normal. Serum carcinoembryonic antigen, carbohydrate antigen 19-9 and prostate specific antigen were within normal range. Cytology examination of the sputum was negative. The contrast-enhanced computed tomography (CT) scan of the chest revealed emphysematous blebs/bullae, enlarged mediastinal lymph nodes and a suspicious $(6 \mathrm{~mm})$ nodule in the upper lobe of the right lung. The CT scan of the upper and lower abdomen revealed adrenal glands swelling, nodules near the left kidney, multiple subcutaneous nodules anteriorly and posteriorly around the thorax and the abdomen as well as enlarged inguinal lymph nodes (Figure 1).

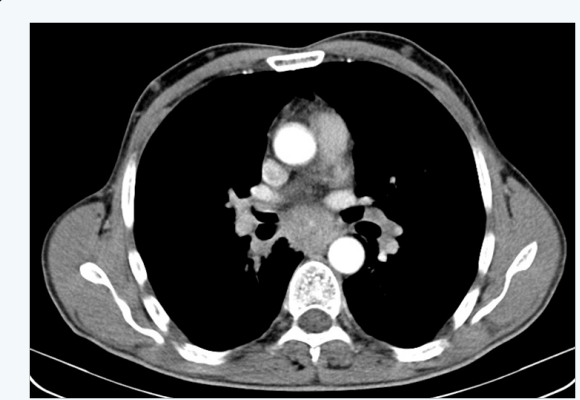

(1a)

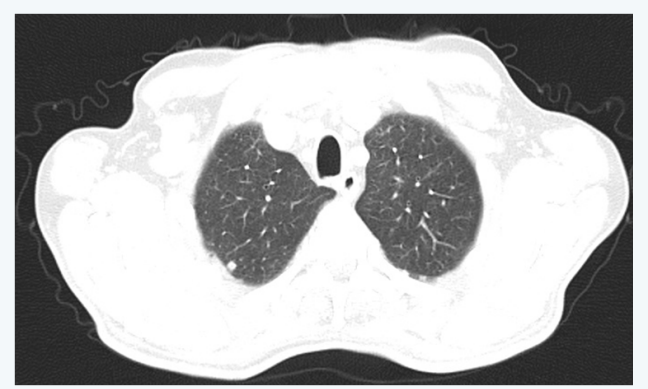

(1b)

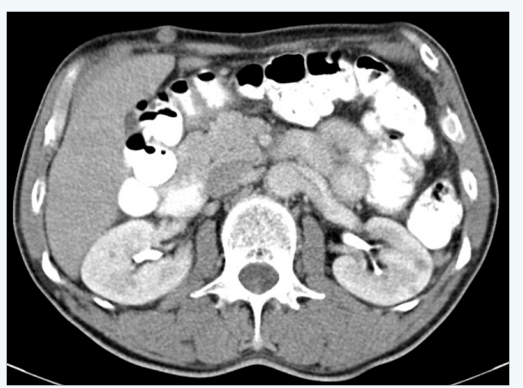

(1c)

Figure 1a: Thorax CT (post enhancement) at the time of diagnosis showing multiple subcutaneous nodules anteriorly and posteriorly around the thorax.

Figure 1b: Thorax CT (post enhancement, lung window) at the time of diagnosis showing a small nodule $(0.6 \mathrm{~cm})$ in the right upper lobe.

Figure 1c: Abdomen CT (post enhancement) at the time of diagnosis showing adrenal glands swelling, nodules near the left kidney and multiple subcutaneous nodules anteriorly and posteriorly around the abdomen.

Magnetic resonance imaging (MRI) of the brain revealed multiple and diffuse pathological supra- and infratentorial lesions $(1.5 \mathrm{~cm}$ max. diameter) of the brain parenchyma (Figure 2$)$. With no primary site of cancer identified, the patient underwent an excisional biopsy of a left inguinal lymph node. Histopathology was suggestive of small cell carcinoma probably originating from the lung. The IHC stains for TTF1, CK7 and synaptophysin were positive and also revealed high expression of Ki-67 (>80\%). In order to find the primary lesion diagnosed by biopsy of the inguinal lymph node, a positron emission tomography (PET) scan was performed. This showed increased tracer uptake in multiple node groups over and under the diaphragm (SUVmax 10 in mediastinum), in bones, bone marrow, pleura, mesentery, adrenal glands (SUV max 13) as well as the subcutaneous tissue of the whole body (Figure 3).

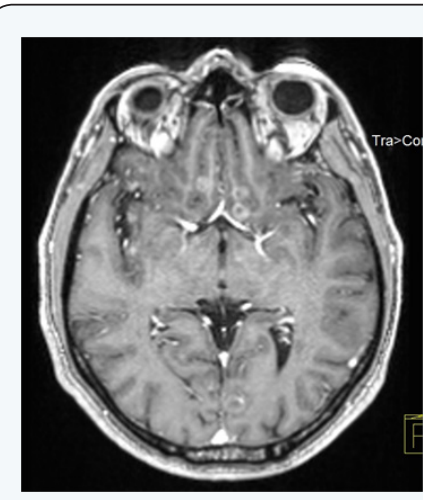

(2a)

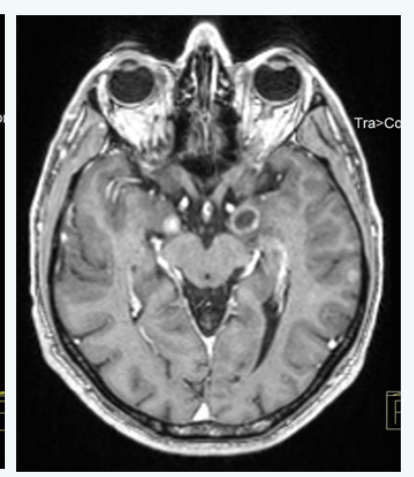

(2b)

Figure 2a, b: MRI of the patient's brain (axial view, T1 weighted post contrast) at the time of diagnosis showing multiple and diffuse pathological supra- and infratentorial lesions $(1.5 \mathrm{~cm}$ max. diameter) of the brain parenchyma.

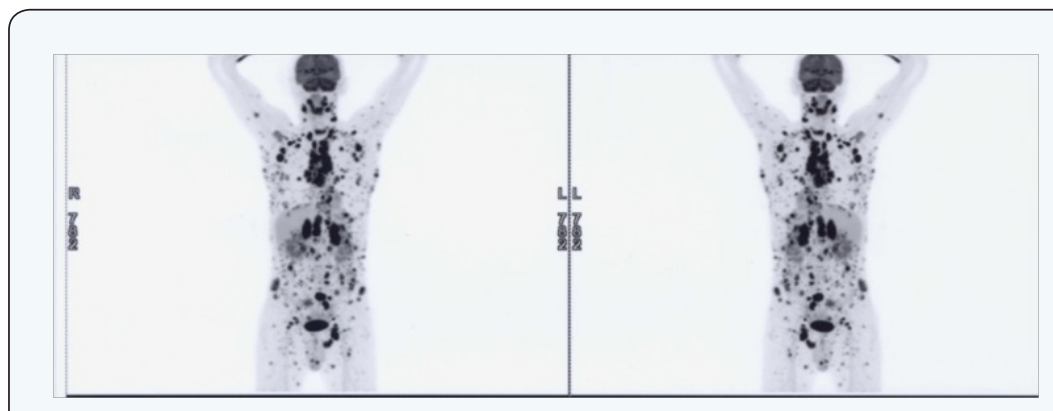

(3a)

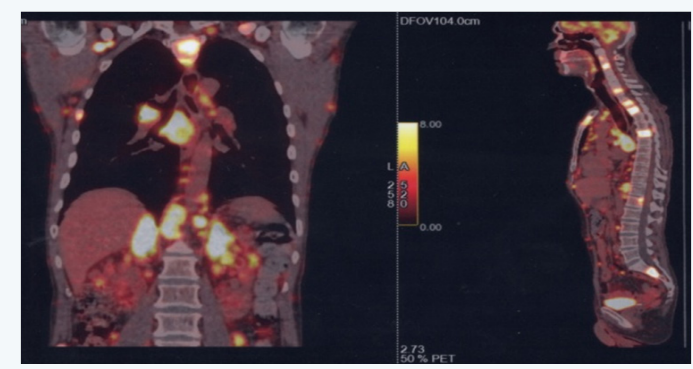

(3b)

Figure 3a, b: FDG-PET scan at the time of diagnosis showing increased tracer uptake in multiple node groups over and under the diaphragm (SUV max 10 in mediastinum), in bones, bone marrow, pleura, mesentery, adrenal glands (SUV max 13) as well as the subcutaneous tissue of the whole body. 
Lungs, liver, spleen and pancreas revealed no tracer uptake. The primary site could not be visualized generating a differential diagnosis between hematological disease (lymphoma) and diffuse metastatic melanoma. Finally, the histopathological diagnosis was reviewed and confirmed by the pathologists of our Hospital. An appropriate battery of immunostains revealed the following immunophenotype: AE1/AE3+, CK8/18+, TTF1+, CD56+, NSE+, synaptophysin++/-, chromogranin--/+, CK7--/+, CK20-, CD45-, S100-, NF-. Cell proliferation index Ki67/MIB1 was high (>80\%). The lymph node metastasis showed morphological and immunohistochemical features of neuroendocrine small cell carcinoma (Figure 4) indicating a possible primary site in the lung. Palliative whole brain radiotherapy was performed in our department delivering 30 Gy in 10 fractions. Significant clinical improvement in terms of memory loss was immediately observed. Chemotherapy was administered as a platinum/ etoposide-based combination schedule with remarkable clinical response immediately. After chemotherapy, the CT imaging study confirmed substantial regression of all metastatic lesions (Figure 5).

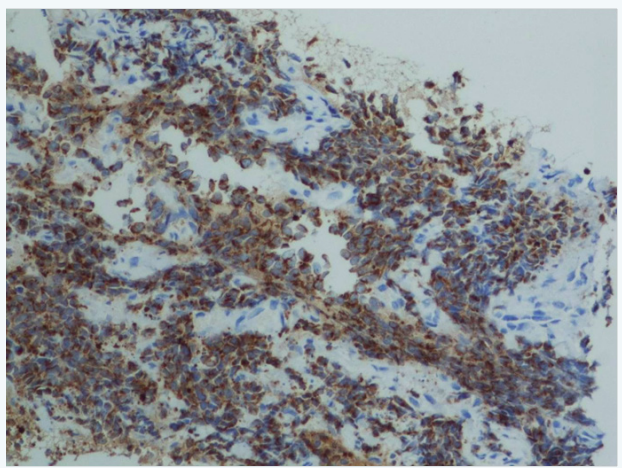

(4a)

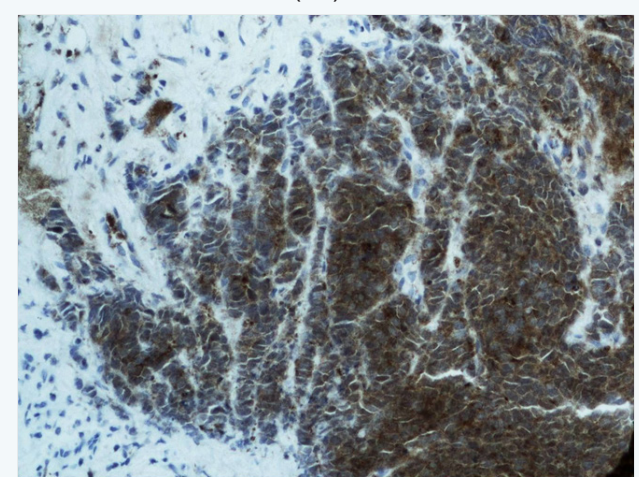

(4c)

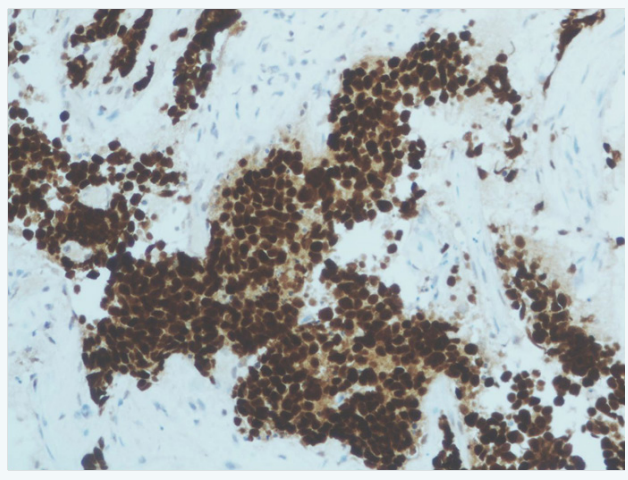

(4b)

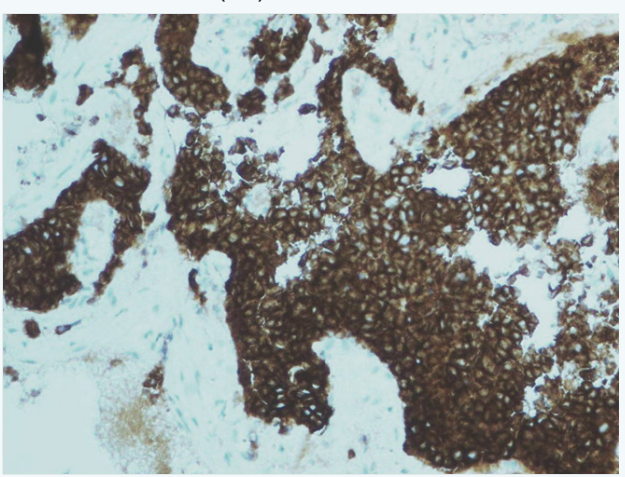

(4d)

Figure 4: On immunohistochemical examination the neoplastic cells stained strongly positive for AE1AE3 (a), TTF1 (b), CD56 (c), and NSE (d) (a-d: IHC x200).

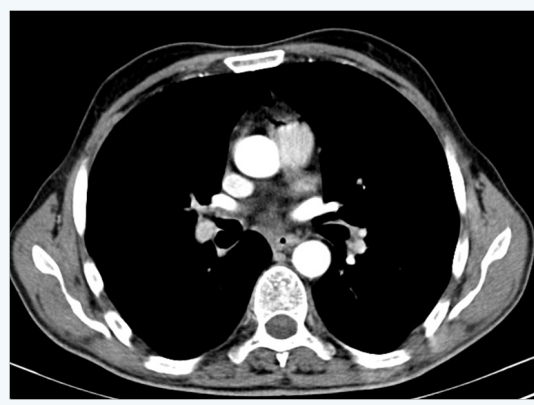

(5a)

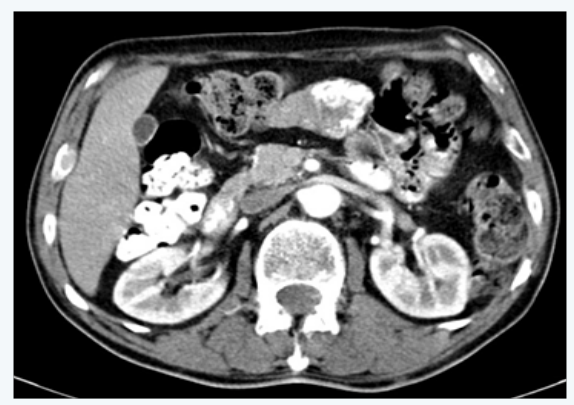

$(5 b)$

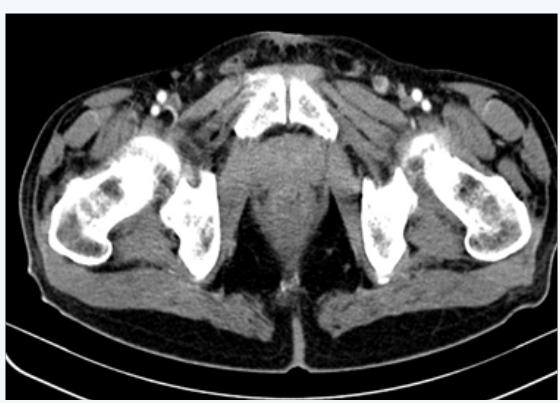

(5c)

Figure 5a: Thorax CT (post enhancement) after chemotherapy showing significant regression of the lesions.

Figure 5b, c: Abdomen and pelvic CT (post enhancement) after chemotherapy showing significant regression of the lesions. 


\section{Discussion}

Our patient suffered from cough, worsening back pain and altered mental status. He clinically presented multiple palpable subcutaneous nodules on the trunk and swelling of the groin lymph nodes. At FDG PET, the lungs, the liver, the spleen and the pancreas revealed no tracer uptake raising the suspicion of hematological disease (lymphoma) or diffuse metastatic melanoma. However, the diagnosis of neuroendocrine small cell carcinoma was established by the characteristic morphology on histology and an IHC neuroendocrine marker pattern (CD56, chromogranin, synaptophysin, TTF1). Patients with small or large cell histology and a history of cigarette smoking should be suspected of having an occult lung primary. Our patient presented widespread metastatic disease, for which no primary site could be detected after clinical examination and extensive investigations.

CT scan revealed no mass lesions, except a small indeterminate nodule in the lung. It is rather unlikely for this to have been the primary site as the nodule was very small and negative on PET-CT. CUP is a diagnosis of exclusion, since many studies did not include other types of tumors such as lymphoma, melanoma and sarcoma, as well as tumors of unusual primary sites. However, since those types of carcinomas often enter the clinical and pathological differential diagnosis, they should be considered $[5,8]$. The diagnostic procedure begins, if necessary, by determining the cell lineage (epithelial, melanocytic, lymphoid, mesenchymal or germinal) with the aid of appropriate IHC markers. The choice of the IHC panel deeply influences subsequent diagnosis. In some cases IHC algorithms can allow the identification of a primary site with adequate accuracy [9].

Metastases of unknown primary origin are divided in two groups: 1) those in which the primary site might be defined at least by their IHC profile; 2) those which really remain 'orphan' even after an appropriate IHC screening [10]. The primary site may either have a slow growth or may possibly become involute and therefore unlikely to manifest itself. In some instances, although the metastatic pattern is often unpredictable, the site of primary origin can be found during lifetime or autopsy. Generally it is a small nodule often localized in the lungs or in the bilio-pancreatic tract [11]. Since the unknown primary mass can be located anywhere in the body, a cross-sectional whole-body imaging modality is the proper method to search for it. Small lesions or pathological changes in normal-sized tissues can be missed by CT and MRI [12]. From this perspective, PET using the radiotracer 18 F-fluoro-2-deoxyglucose is the leading approach, since it provides functional and metabolic information.

Notably, PET/CT imaging is known to have good sensitivity and specificity, mainly in head/neck and lung cancers [13]. Patients with high-grade neuroendocrine tumors are initially responsive to combination chemotherapy and should be considered for a trial of treatment. However, the treatment is approached similarly to extensive stage small cell lung cancer, as these cancers have similar histology. Treatment includes standard chemotherapy used to treat lung cancers, including platinum-based or platinum-taxane combination chemotherapy. This group of patients presented response rates up to $55 \%$ with $20 \%$ complete responders and overall survival of 15 months as well as almost 10-15\% long-term survivors [14].

\section{Conflict of interest}

I have no financial interest or any conflict of interest.

\section{References}

1. Levi F, Te VC, Erler G, Randimbison L, La Vecchia C (2002) Epidemiology of unknown primary tumours. Eur J Cancer 38(13): 1810-1812.

2. Briasoulis E, Pavlidis N (1997) Cancer of unknown primary origin. Oncologist 2(3): 142-152.

3. Mukur Dipi Ray, Shivam Vatsal, Sunil Kumar (2015) Metastatic inguinal lymph nodes with two different histological types in a case of carcinoma of unknown primary site. Journal of Cancer Metastasis and Treatment 1(2): 101-103.

4. Stoyianni A, Pentheroudakis G, Pavlidis N (2011) Neuroendocrine carcinoma of unknown primary: a systematic review of the literature and a comparative study with other neuroendocrine tumors. Cancer Treat Rev 37(5): 358-365.

5. Pavlidis N (2003) Cancer of unknown primary: biological and clinical characteristics. Ann Oncol 14(3): 11-18.

6. Oien KA, Dennis JL (2012) Diagnostic work-up of carcinoma of unknown primary: from immunohistochemistry to molecular profiling. Annals of Oncology 23(10): x271-x277.

7. Spigel DR, Hainsworth JD, Greco FA (2009) Neuroendrocrine carcinoma of unknownprimary site. Semin Oncol 36(1): 52-59.

8. Massard C, Loriot Y, Fizazi K (2011) Carcinomas of an unknown primary origin-diagnosis and treatment. Nat Rev Clin Oncol 8(12): 701-710.

9. Greco FA (2012) Cancer of Unknown Primary Site: Evolving Understanding and Management of Patients. Clin Adv Hematol Oncol 10(8): 518-524.

10. Greco FA, Hainsworth JD (2011) Cancer of unknown primary. In: De Vita TV, et al. (Eds.), Cancer Principles and Practice of Oncology. ( $9^{\text {th }}$ edn), Wolters, Kluwer/Lippincott, Williams and Wilkins, Philadelphia, USA. pp. 2033-2051.

11. van de Wouw AJ, Jansen RL, Speel EJ, Hillen HF (2003) The unknown biology of the unknown primary tumors: a literature review. Ann Oncol 14(2): 191-196.

12. Pavlidis N (2007) Forty years experience of treating cancer of unknown primary. Acta Oncol 46(5): 592-601.

13. Sève P, Billotey C, Broussolle C, Dumontet C, Mackey JR (2007) The role of 2-deoxy-2-[F-18]fluoro-D-glucose positron emission tomography in disseminated carcinoma of unknown primary site. Cancer 109(2): 292-299.

14. Pavlidis N, Khaled H, Gaafar R (2015) A mini review on cancer of unknown primary site: A clinical puzzle for the oncologists. J Adv Res 6(3): 375-382. 